\title{
TRIGGER AND READOUT ELECTRONICS FOR THE PHASE-I UPGRADE OF THE ATLAS FORWARD MUON SPECTROMETER
}

\section{PARIS MOSCHOVAKOS} on behalf of ATLAS Muon Collaboration

National Technical University of Athens, Brookhaven National Laboratory MOCAST, 4-6 MAY 2017, THESSALONIKI, GREECE 


\section{OUTLINE}

- Introduction

- New Small Wheel electronics

- Data Path

- Trigger Path

- Prototype boards \& On detector placement

- Detector Control System - SCA ASIC

- Vertical Slice integration

- Summary 
INTRODUCTION 


\section{NEW SMALL WHEEL MOTIVATION AND TRIGGER PLANS}

\section{Small Wheels}

- Present detectors:

- Cathode Strip Chambers - CSC

- Monitored Drift Tubes - MDT

- Thin Gap Chambers - TGC

- Not efficient in high rates (maximum $20 \mathrm{KHz} / \mathrm{cm}^{2}$ )

- $90 \%$ of the current End-cap muon triggers are fakes

\section{New Small Wheels}

- Detectors:

- Micromegas - MM

- small Thin Gap Chambers - sTGC

- A set of precision tracking and trigger detectors able to work at high rates

- Will provide online high quality ( $\sigma \theta \sim 1 \mathrm{mrad})$ IP pointing segments

- Significant reduction of fake Level-1 muon triggers

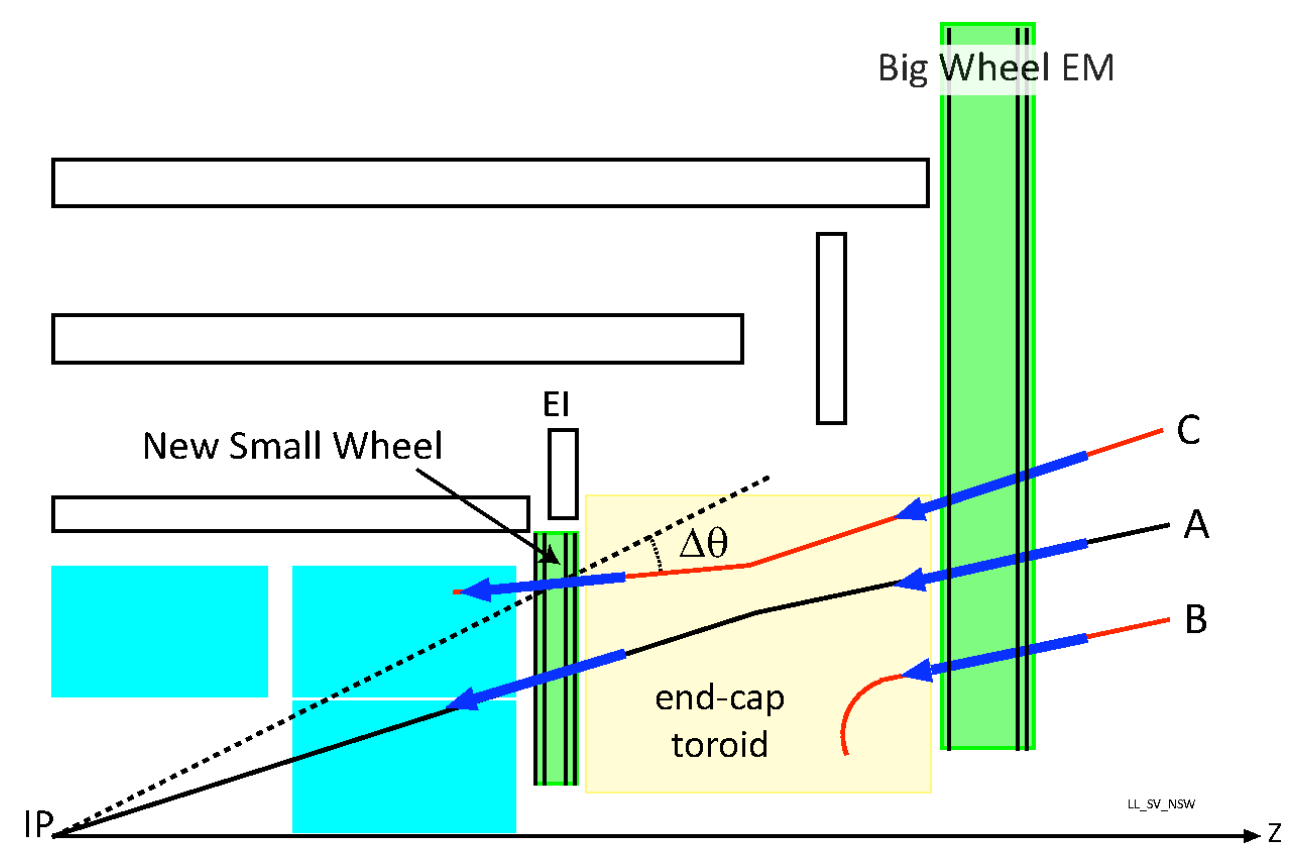

\section{Requirements}

- Phase-1 (2019-2020)

- Level-1 accept @ 100 KHz

- Phase-2 (2024-2026)

- One level-0 accept @ $1 \mathrm{MHz}$

- Dual level: Level-0 @ 1 MHz \& Level-1 @ $400 \mathrm{KHz}$

- Dual level : Level-0 @ 4MHz \& Level-1 @ $600 \mathrm{KHz}$ 


\section{NEW SMALL WHEEL ELECTRONICS}




\section{ELECTRONICS GLOBAL SCHEME}

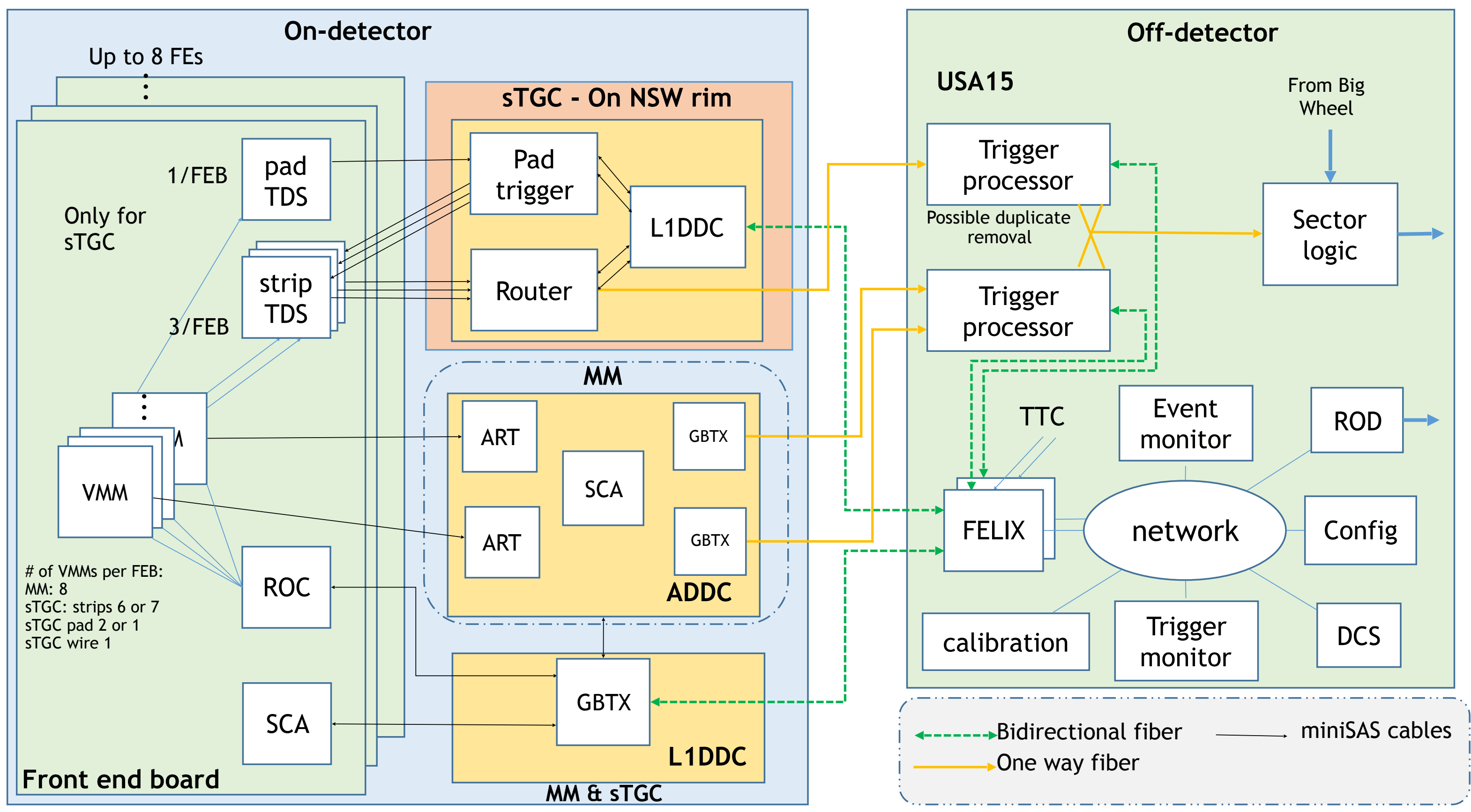

- Radiation tolerant ASICs

- Slow Control Adapter (SCA)

- Read Out Controller (ROC)

-Address Real Time (ART)

- GigaBit Transceiver (GBTX)

- Trigger Data Serialiser (TDS)

-VMM (amplifier, discriminator, shaper)
- Front-End LInk eXchange (FELIX) Interface network

- Timing Trigger \& Control (TTC)

- Art Data Driver Card (ADDC)

- Read Out Device (ROD)

- Detector Control System (DCS)

- Pad trigger and router use commercial FPGAs 


\section{MICROMEGAS AND STGC READOUT PATH}

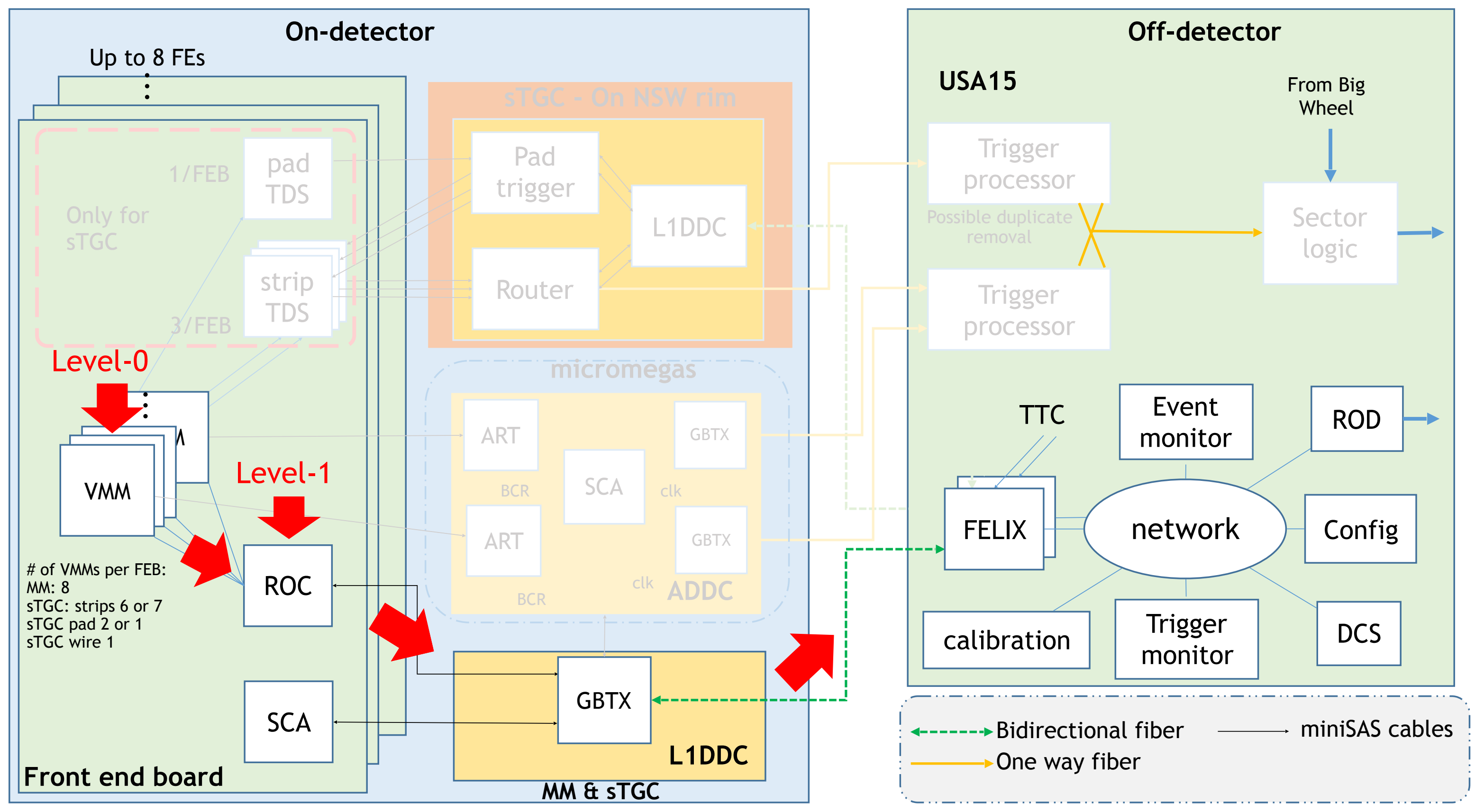

- MMs - 2.097.152 channels

- sTGC - 331.744 channels

- From FELIX

- Time trigger and control signals

- Configuration data
- To FELIX

- Level-1

- Monitoring data

- 2 separate L1DDC (sTGC \& Micromegas)

- Upon a level-0 accept data to ROC

- Upon a level-1 accept data to FELIX 


\section{MICROMEGAS TRIGGER PATH}
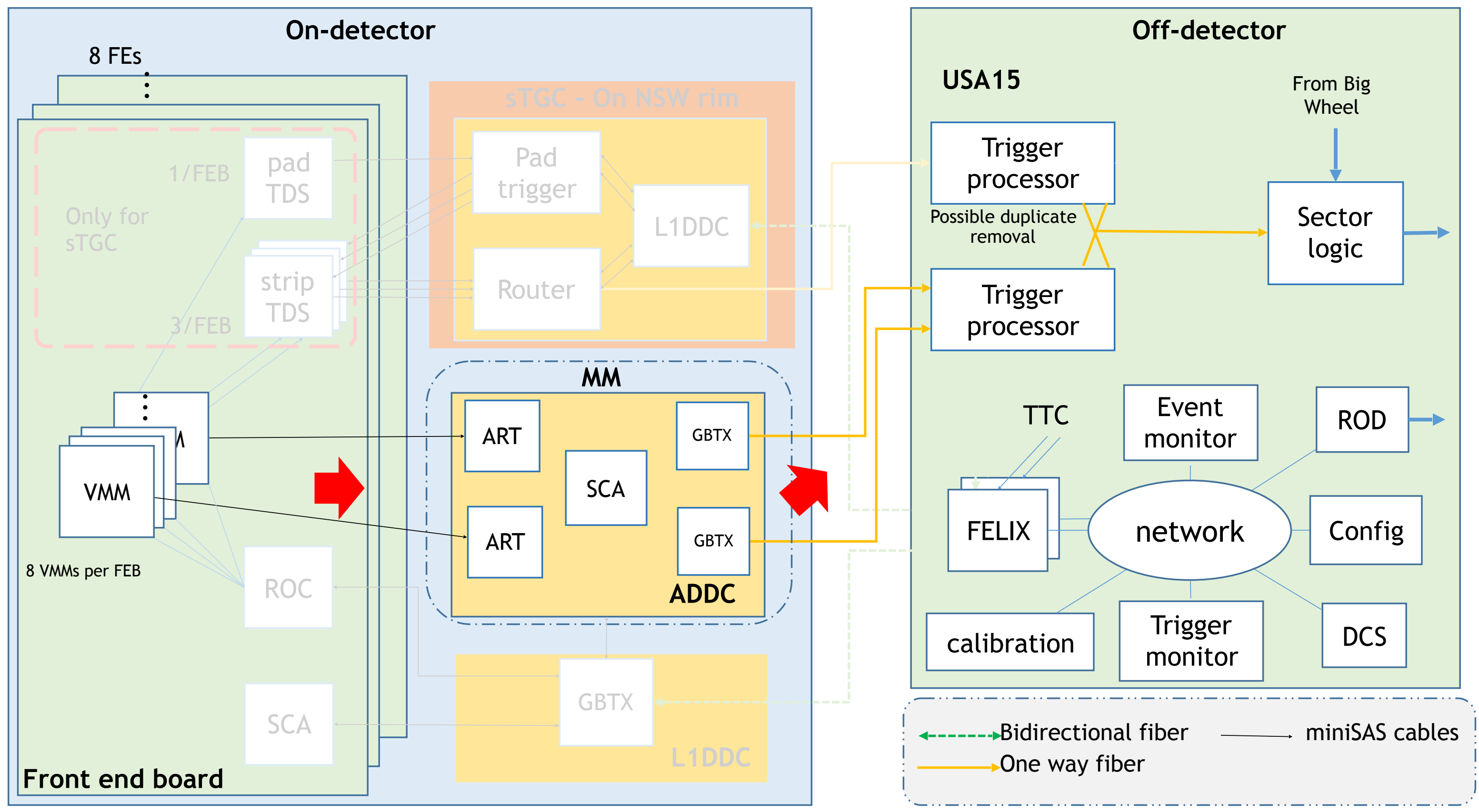

- Channel address of the first hit from each VMM is sent to the ADDC boards

- 1 ART ASIC has 32 inputs (coming from 4 front end boards)

- A priority based hit selection is implemented in real time

- Selected data are sent to trigger Processor 


\section{STGC TRIGGER PATH}

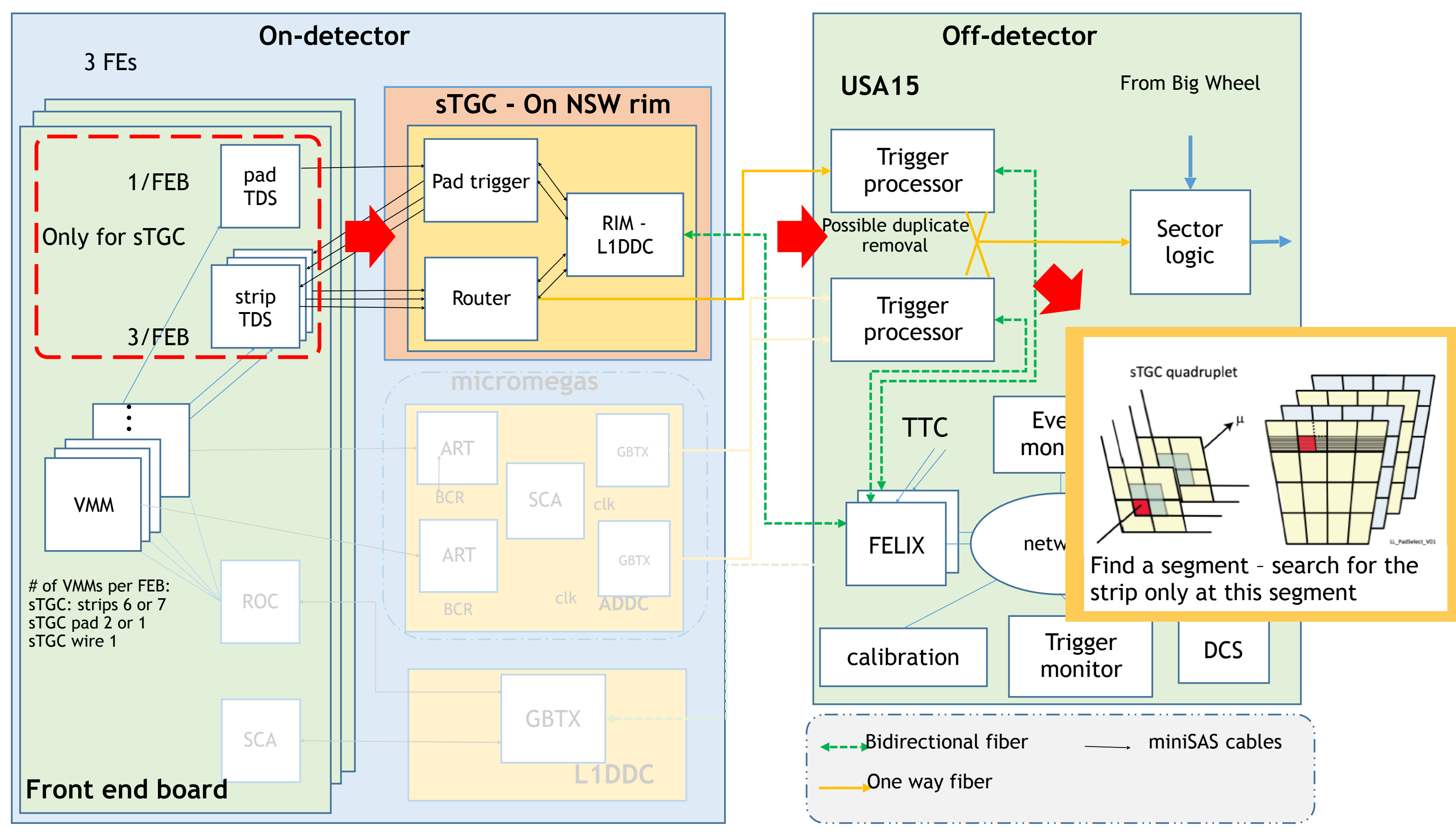

- Time \& amplitude are sent to the pTDS

- Strips are buffered and tagged with the BCID *

- Pad trigger logic identifies a track

- The candidate geometrical coordinates \& corresponding BCID are sent to STDS
- sTDS transmits the strip charges, BCID, band-ID, and $\varphi$-ID Signals are sent to track finding processors where centroids and track segments are calculated

- Finally, sector logic combines data with candidate tracks from the Big Wheel

- RIM-L1DDC for configuration of the RIM electronics 


\section{PROTOTYPE BOARDS \& ON DETECTOR PLACEMENT}




\section{PROTOTYPE BOARDS}

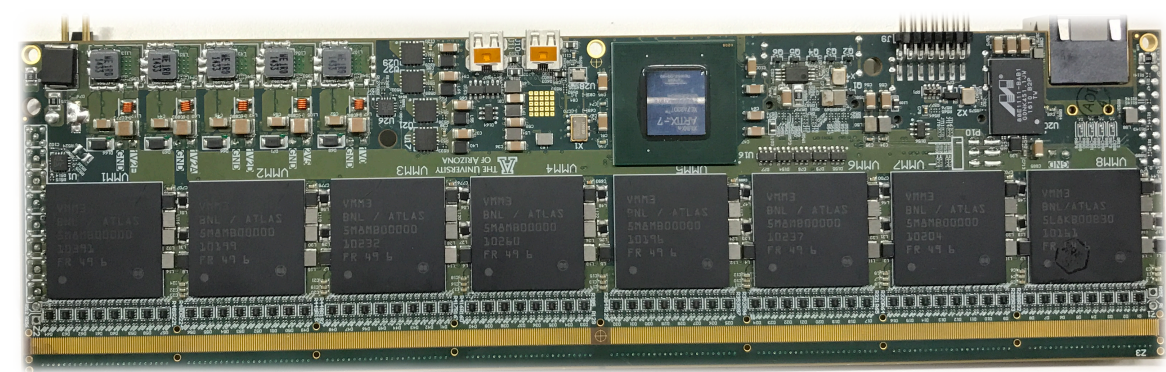

MMFE8

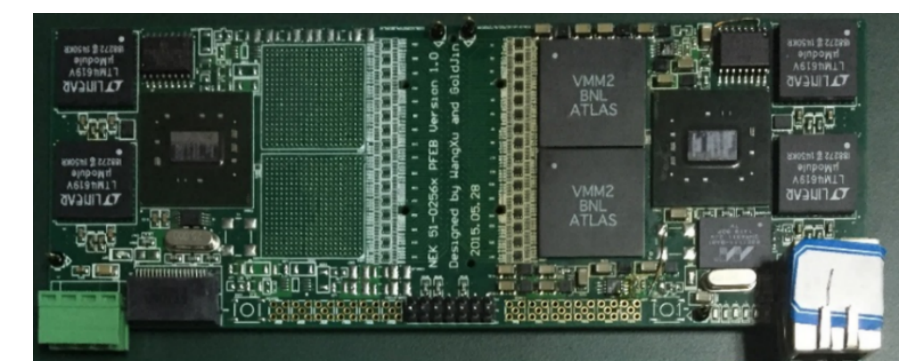

pad\&wire FE

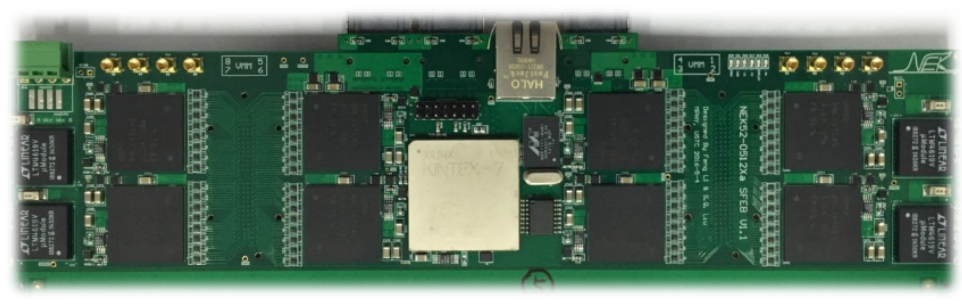

strip FE

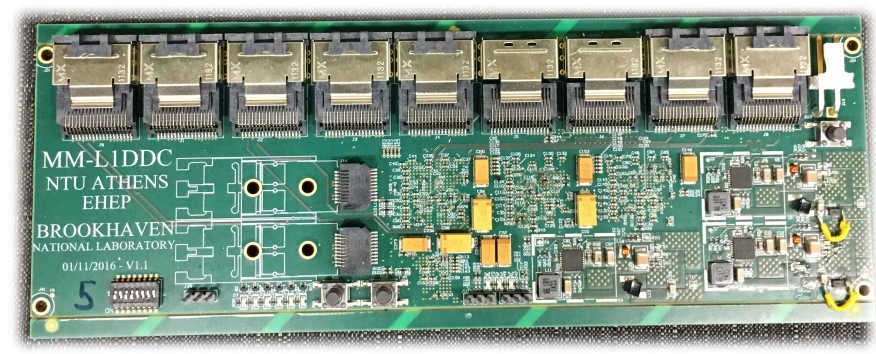

L1DDC

- Most of the prototype boards have already been fabricated

- Using commercial FPGAs until all ASICs are fabricated and tested

- Mini-FELIX and trigger processor running on evaluation boards

- MMFE8

- New prototype with VMM3 is already fabricated and under testing

- Next prototype with SCA and ROC will be submitted later this year

- L1DDC

- Prototype 2 was fabricated. A new minor revision will be needed.

- L1DDC and ADDC new prototypes use only radiation and magnetic tolerant components based on final requirements (dimensions, rate, components)

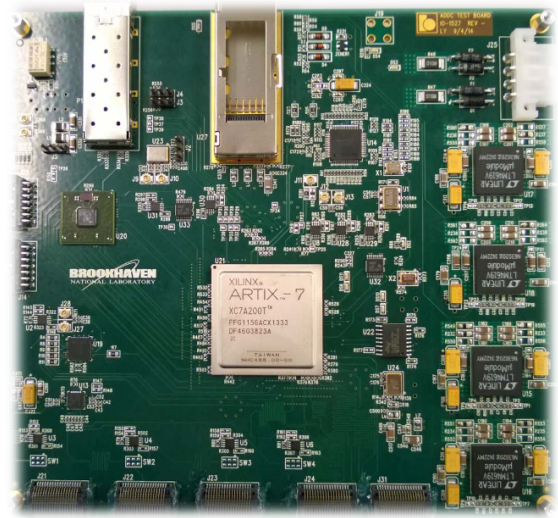

ADDC

Mini-FELIX

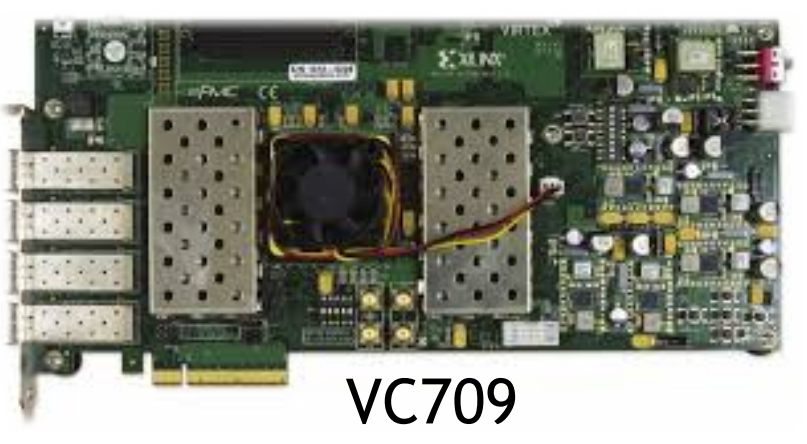

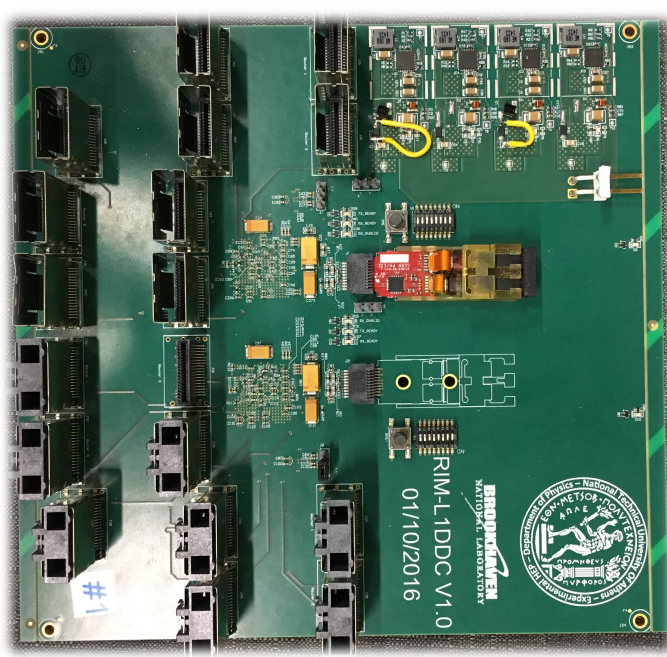

RIM-L1DDC

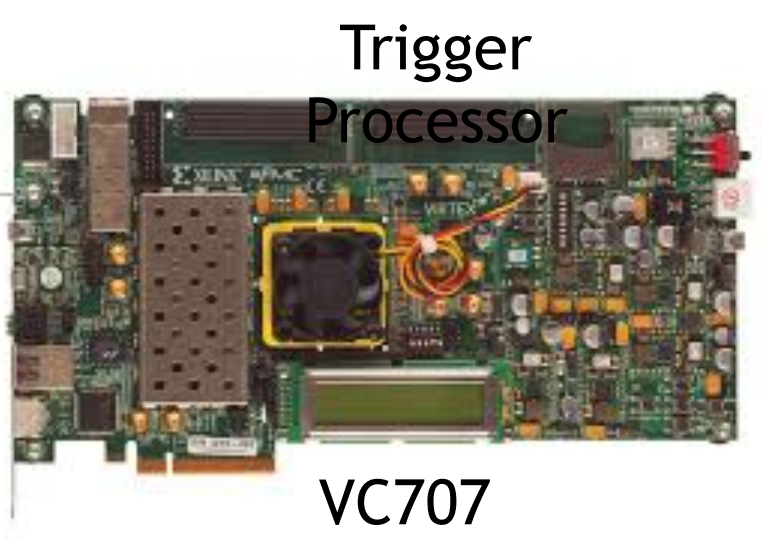

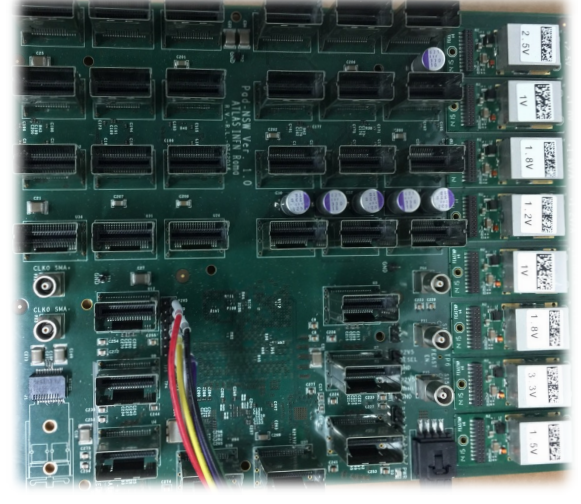

PAD trigger

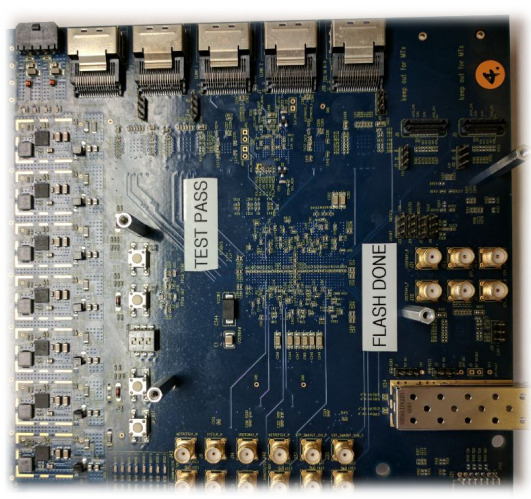

Router

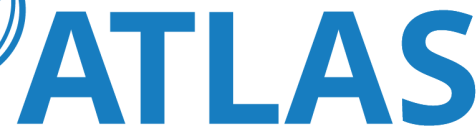
EXPERIMENT 


\section{ELECTRONIC BOARD PLACEMENT ON MM DETECTORS}

- FEs will be placed radially on both sides of each plane

- L1DDC and ADDC boards will be placed only on the $1^{\text {st }}$ and $4^{\text {th }}$ plane of each wedge (4 planes)

- 1 L1DDC \& 1 ADDC will serve the 8 FE located on each side of a plane and will be placed on the centre to minimise the cable length

- 512 L1DDC

- 512 ADDC

- 4096 MMFE8
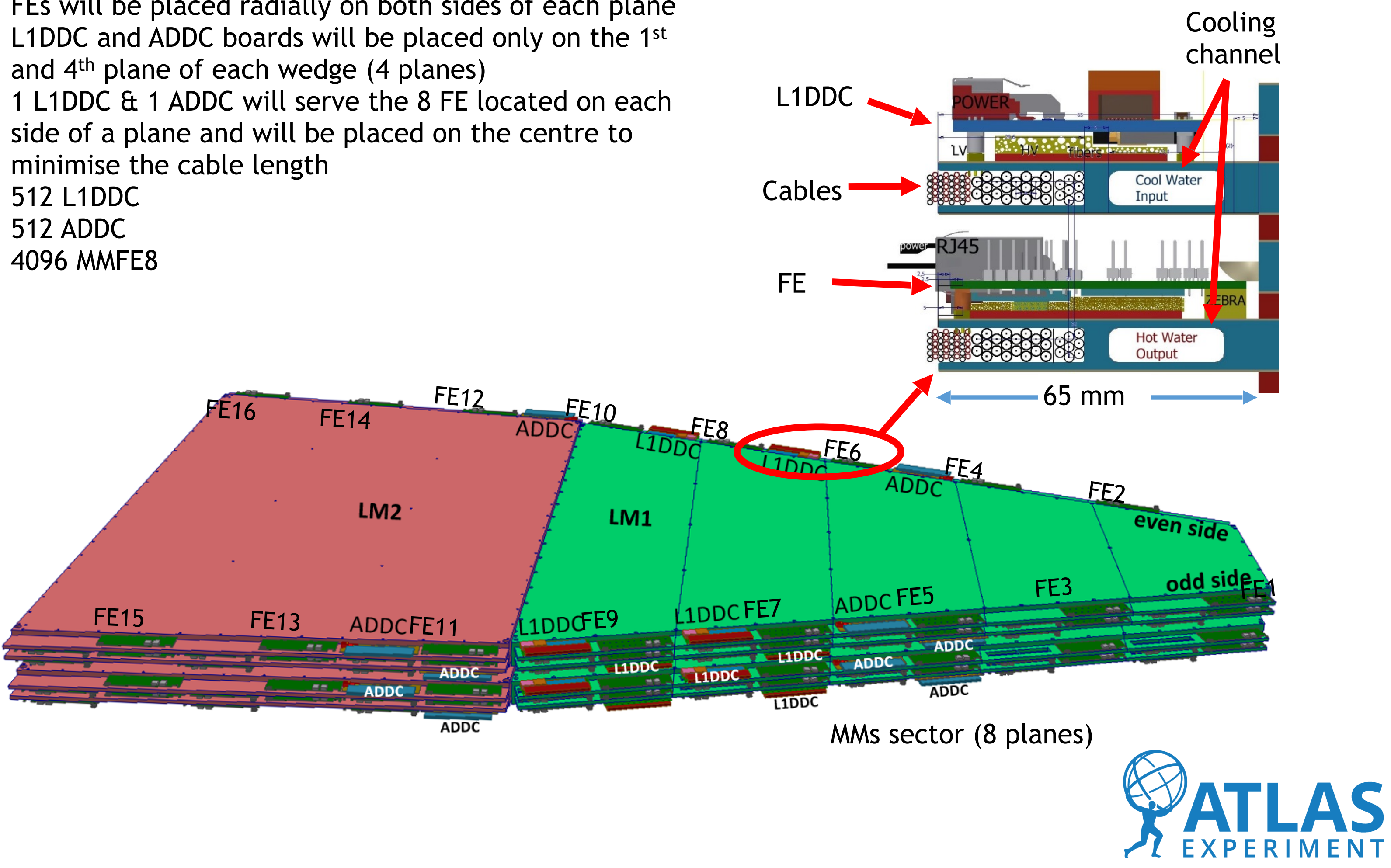


\section{ELECTRONIC BOARD PLACEMENT ON STGC DETECTORS}

- FEs radially on both sides of each plane

- Faraday cage will be used for the sTGC $\mathrm{FE}$ to isolate the sensitive electronics

- L1DDC will be placed on the upper part close to the rim for accessibility reasons

- 1 L1DDC will serve 3 front ends of each plane

- $768 \mathrm{sFE}$

- 768 p\&wFE

- 512 L1DDC
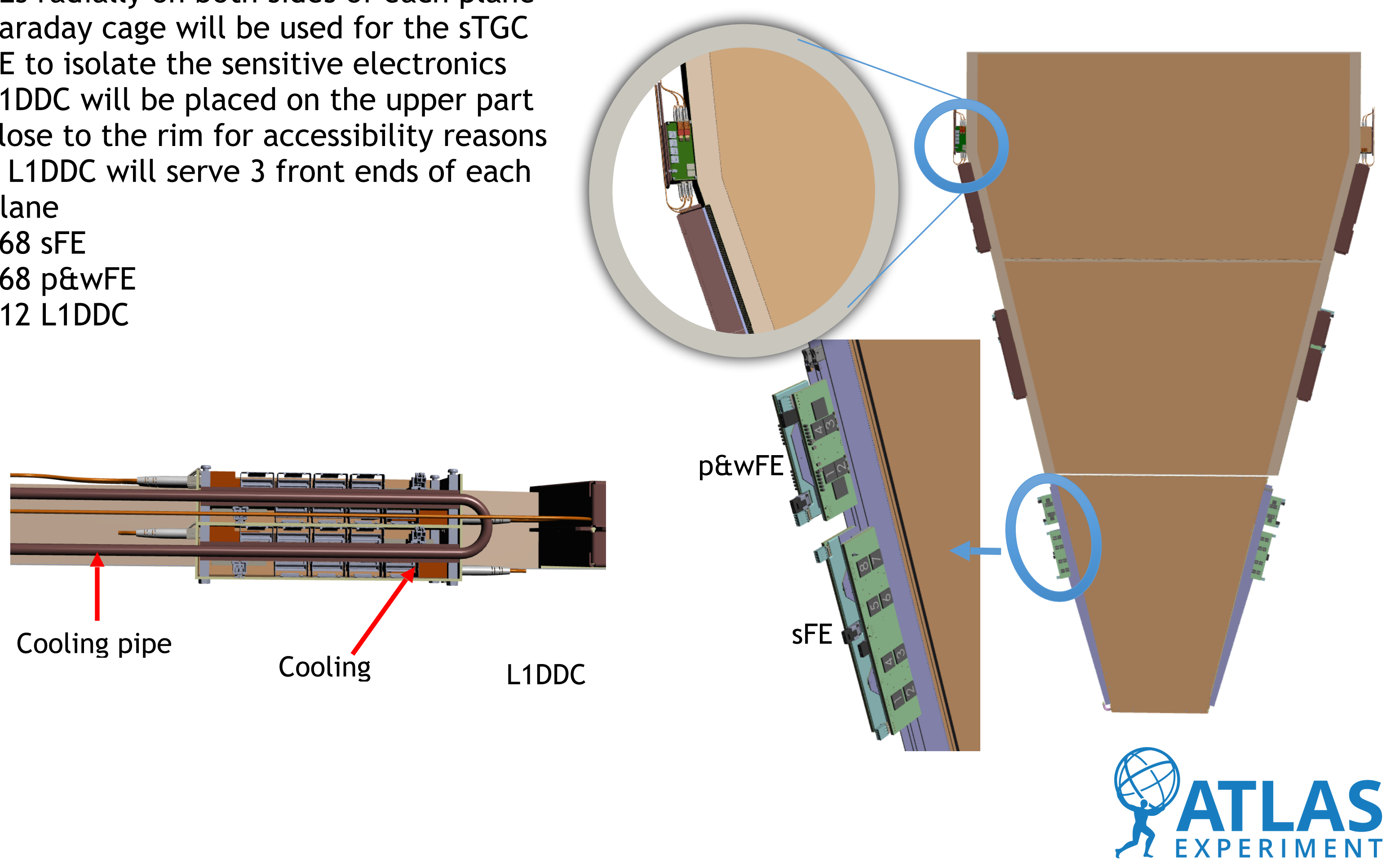


\section{RIM ELECTRONICS}

- 1 RIM box per sector

- Commercial boxes and electronics will be used

- 32 RIM-L1DDC

- 32 Pad trigger boards

- 256 Routers boards
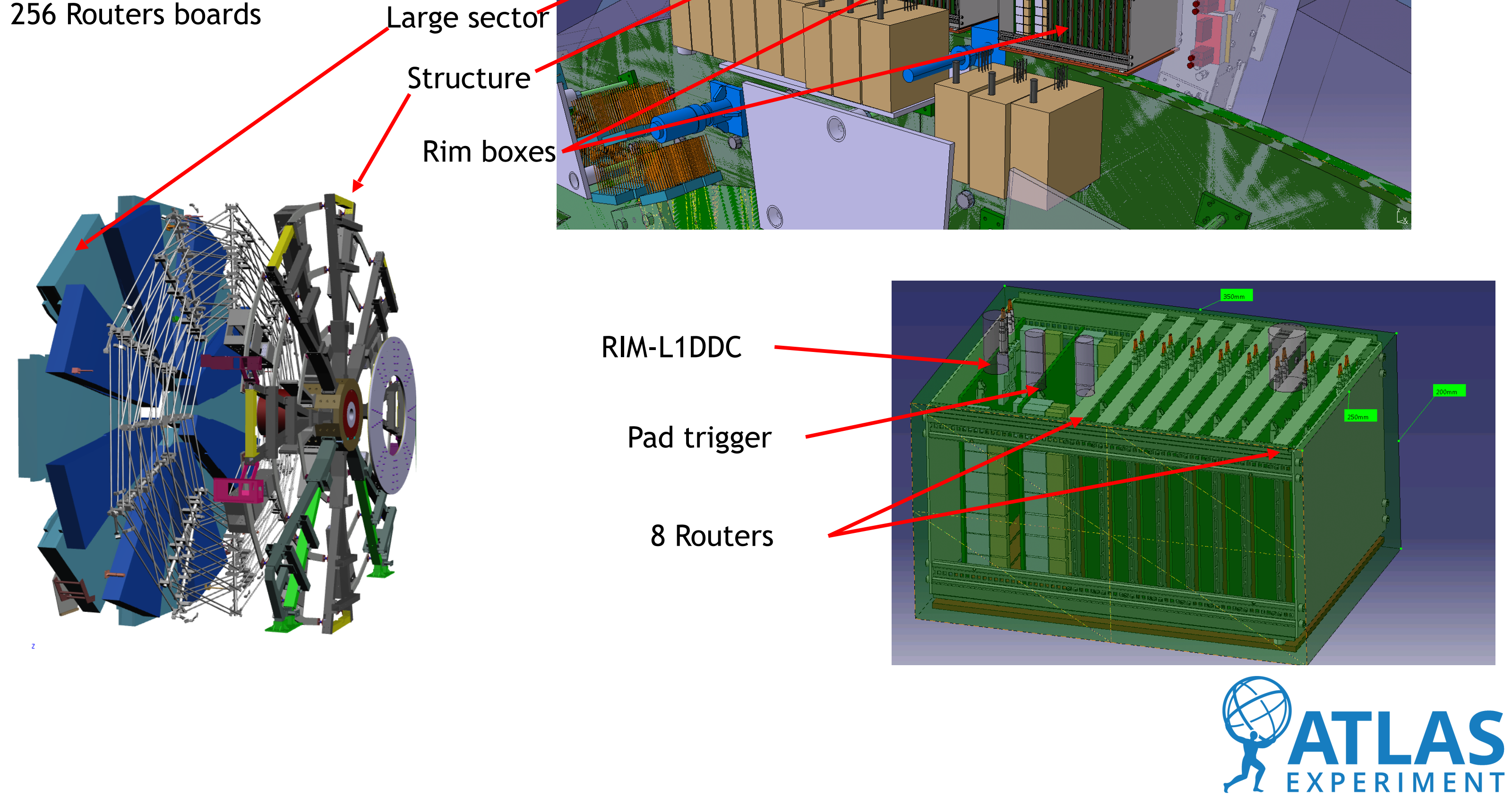


\section{NSW \\ DETECTOR CONTROL SYSTEM}




\section{SCA (SLOW CONTROL ADAPTER) ASIC}

- SCA - What is it?

SCA is a radiation tolerant ASIC for slow control

- 16 independent I2C master serial bus channels (programmable transfer rates $100 \mathrm{KHz}, 200 \mathrm{KHz}$, $400 \mathrm{KHz}, 1 \mathrm{MHz}$ )

- 1 SPI serial bus master channel with 8 individual slave select lines (programmable transaction length up to $128 \mathrm{bits}$, programmable rate $156 \mathrm{KHz}$ to $20 \mathrm{MHz}$ )

-1JTAG serial bus master channel (programmable transaction length up to 128 bits, programmable rate up to $20 \mathrm{MHz}$ )

- 1 Parallel Interface Adapter channel featuring 32 General Purpose I/O line (GPIO) (programmable as input/output, can generate interrupt requests)

- 1 ADC channel include 31 analog inputs and an embedded temperature sensor (12-bit, input range $0.0 \mathrm{~V}$ to $1.0 \mathrm{~V}$ )

- 4 independent digital to analog converter (DAC) channels (8-bit resolution, output range 0.0V - 1.0V)
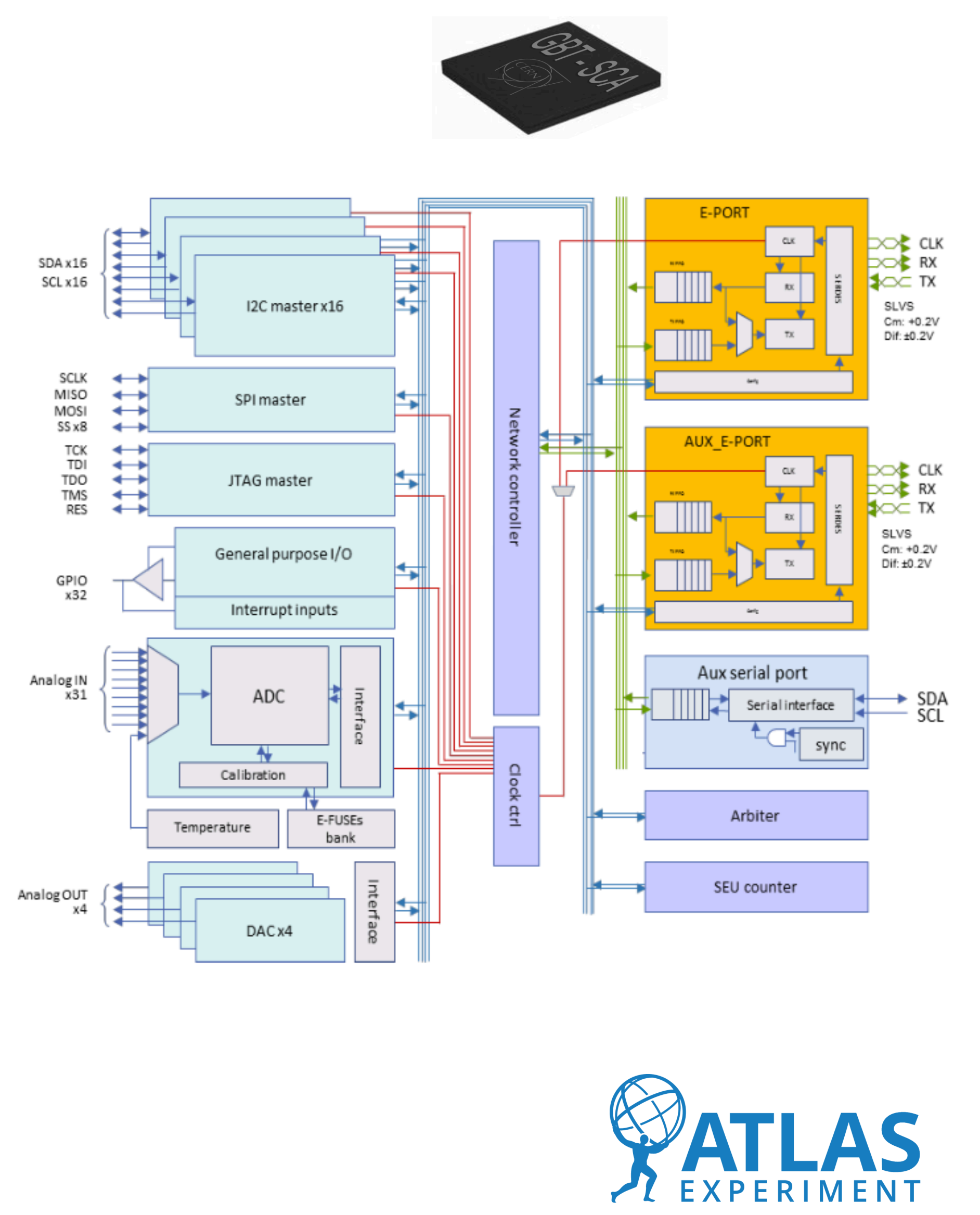


\section{ON-DETECTOR DCS}

\section{TWO INDEPENDENT CONTROL PATHS}

DCS Back-End

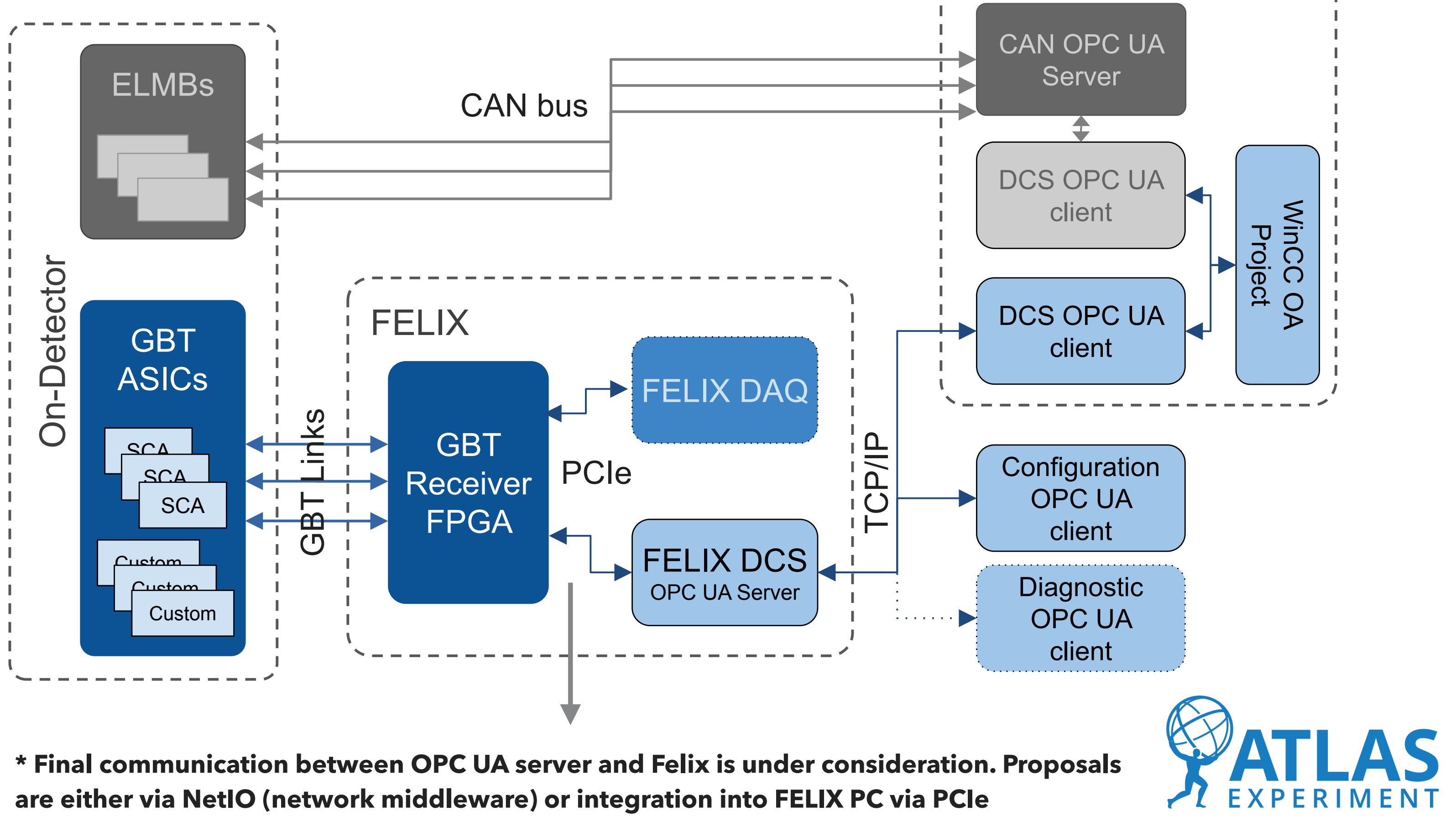




\section{SCA OPC UA SERVER}

- What is it? - Entry point to access SCA via FELIX

- Provides a high level abstraction to the SCA

- i.e. you read Celsius degrees, Volts without knowing the SCA communication protocol

- Establishes timing and synchronisation within SCA(s)

- Supports multiple SCAs within one instance

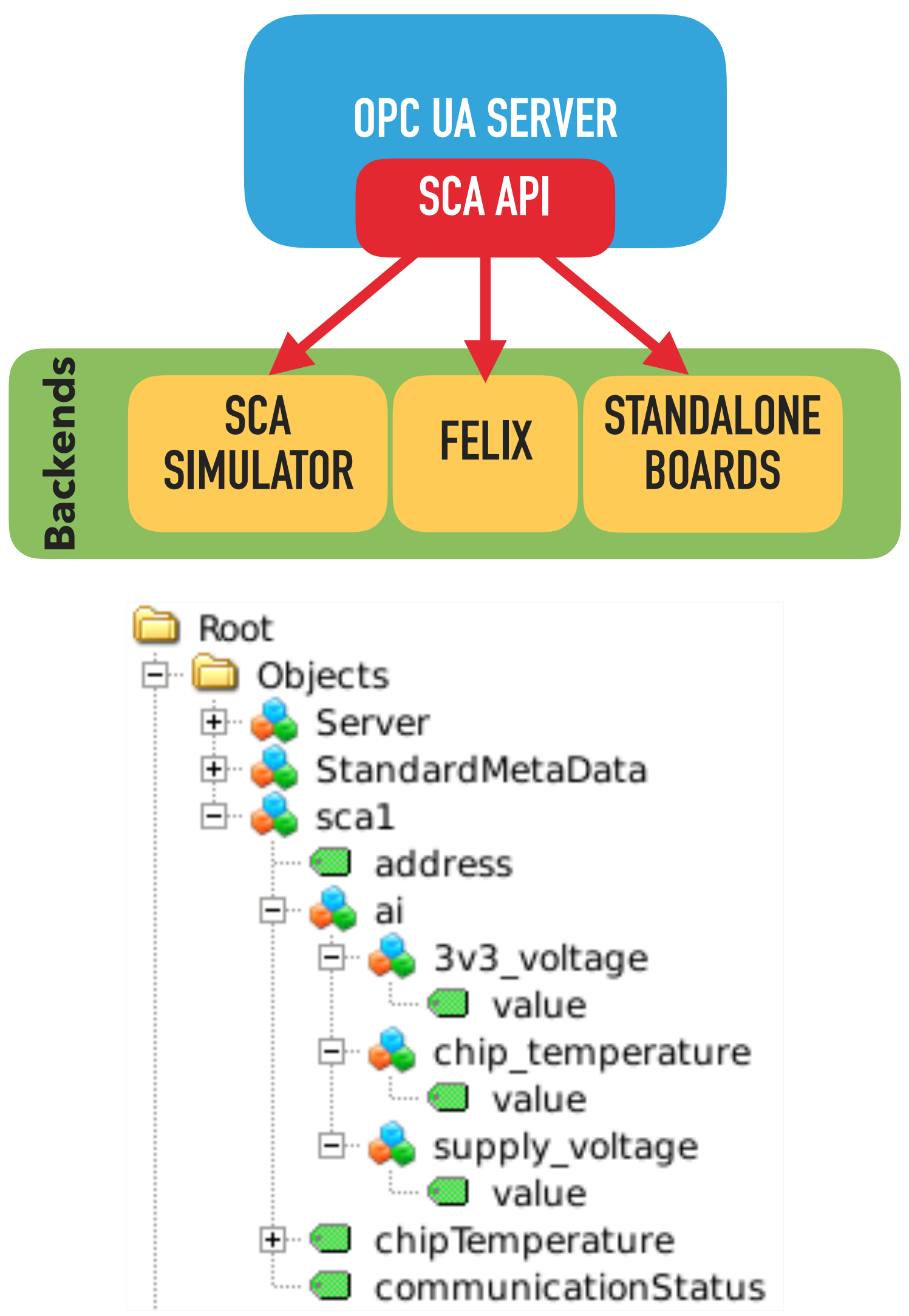




\section{VERTICAL SLICE INTEGRATION}




\section{VERTICAL SLICE RACK AND INTEGRATION WEEKS}

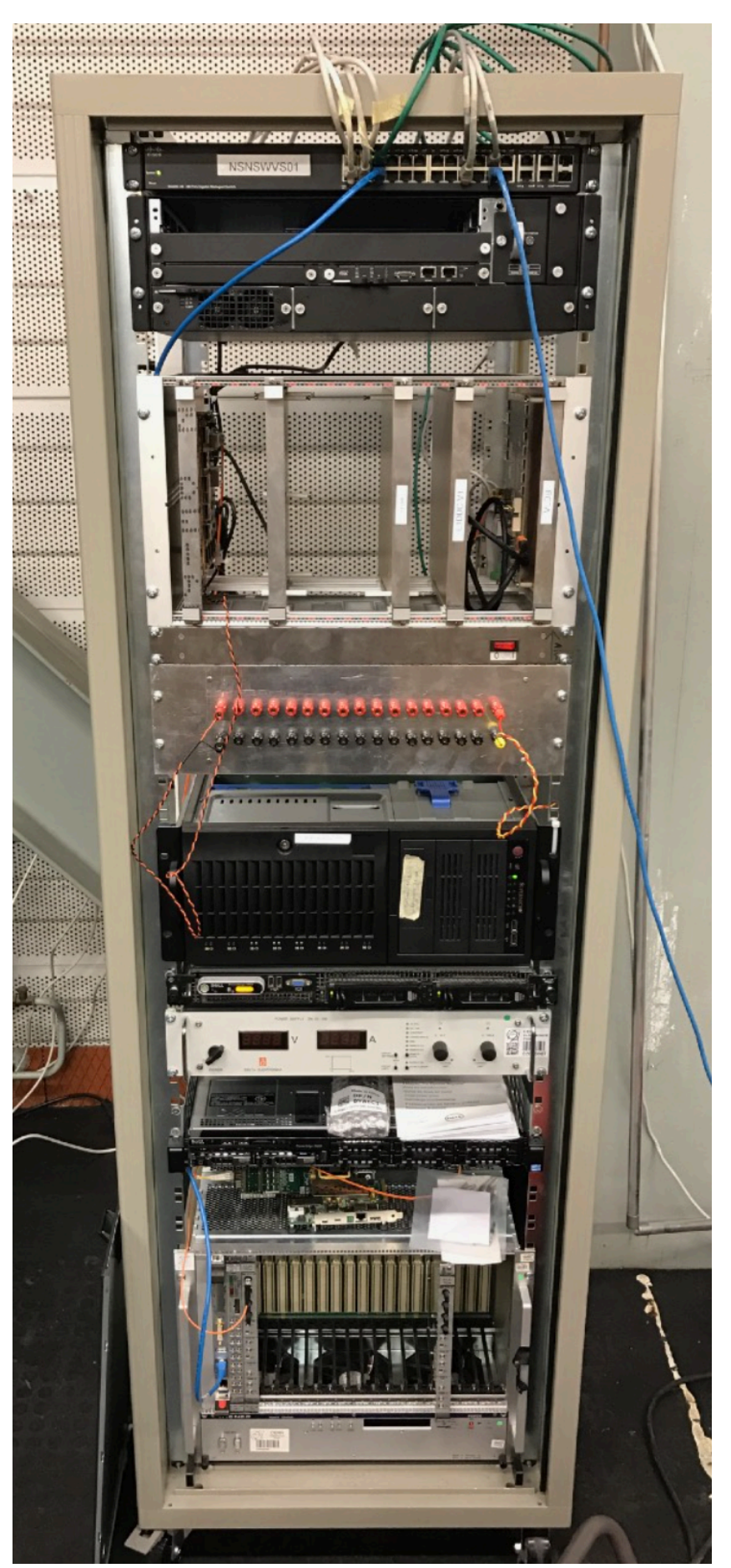

- Switch on CERN network

- ATCA shelf

- Electronics crate

- LV power distribution

- FELIX

- Development machine

- Power supply

- ROD software server

- VME crate, TTC vi/vx - SBC

\section{IN ACTION PHOTOS}

- We had $\sim 14$ groups hosted during the integration weeks

- $\quad 35$ people in each of the weeks (we had to double the space of the lab)
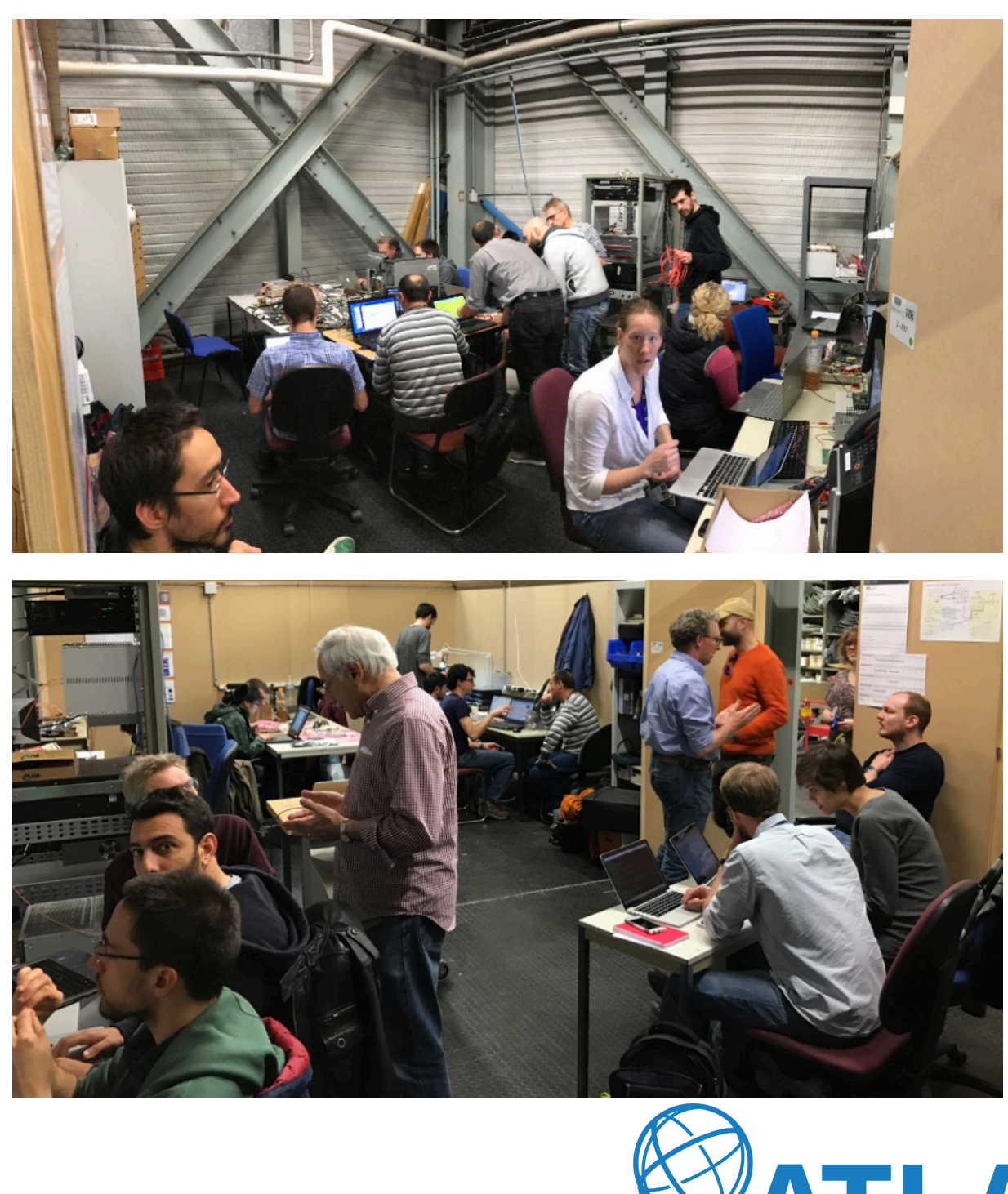


\section{VERTICAL SLICE INTEGRATION ACHIEVEMENTS}

- TTC distribution

- We had established a setup with a FELIX firmware version in which the BC clock was driven by the TTC. This was proven to be stable. We were able to distribute this clock to the FE boards and the trigger boards through L1DDC (RIM electronics as well).

- Various trigger patterns were sent through TTC (L1A) to the MMFE8 through FELIX.

- Data path

- Data communication (both TX and RX) through FELIX has been established, up to 80Mbps. We were able to readout real data coming from the VMM ASICs.

- We have acquired real data that were driven in a loopback and be available to the SROD machine we use for developments.

- Configuration - SCA

- Demonstration of the control of the GPIO on the Router board and the Pad trigger board (RIM electronics) through slow control elinks (via OPC-UA server - client).

- Demonstration of the ADC readout (internal temperature monitor or external input voltage) in WinCC-OA with an ADC trend (change voltage level via potentiometer) from demo board or actual NSW boards.

- Configuration of the GBTX on the L1DDC boards using the IC channel through FELIX.

- GBTX - SCA communication on the L1DDC board. The SCA was able to receive and respond to FELIX.

- Ability to control the SPI channel. Actual configuration of the VMM3 on the USTC SCA demonstrator board through FELIX and GBTx. Milestone for the NSW. 
SUMMARY 


\section{SUMMARY}

- NSW electronics will be able to cope with the high data rates of the HL-LHC

- On detector electronics will use only radiation and magnetic tolerant components

- Off detector electronics and middleware are completing the last pieces of the scheme

- Integration weeks proved that the goal is achievable

- The Slow Control chain with the OPC UA is advancing well

- Larger portions of the chains were demonstrated lately

- Work is still needed to finalise the last prototypes and demonstrate the final vertical slice 
THANK YOU FOR YOUR ATTENTION!

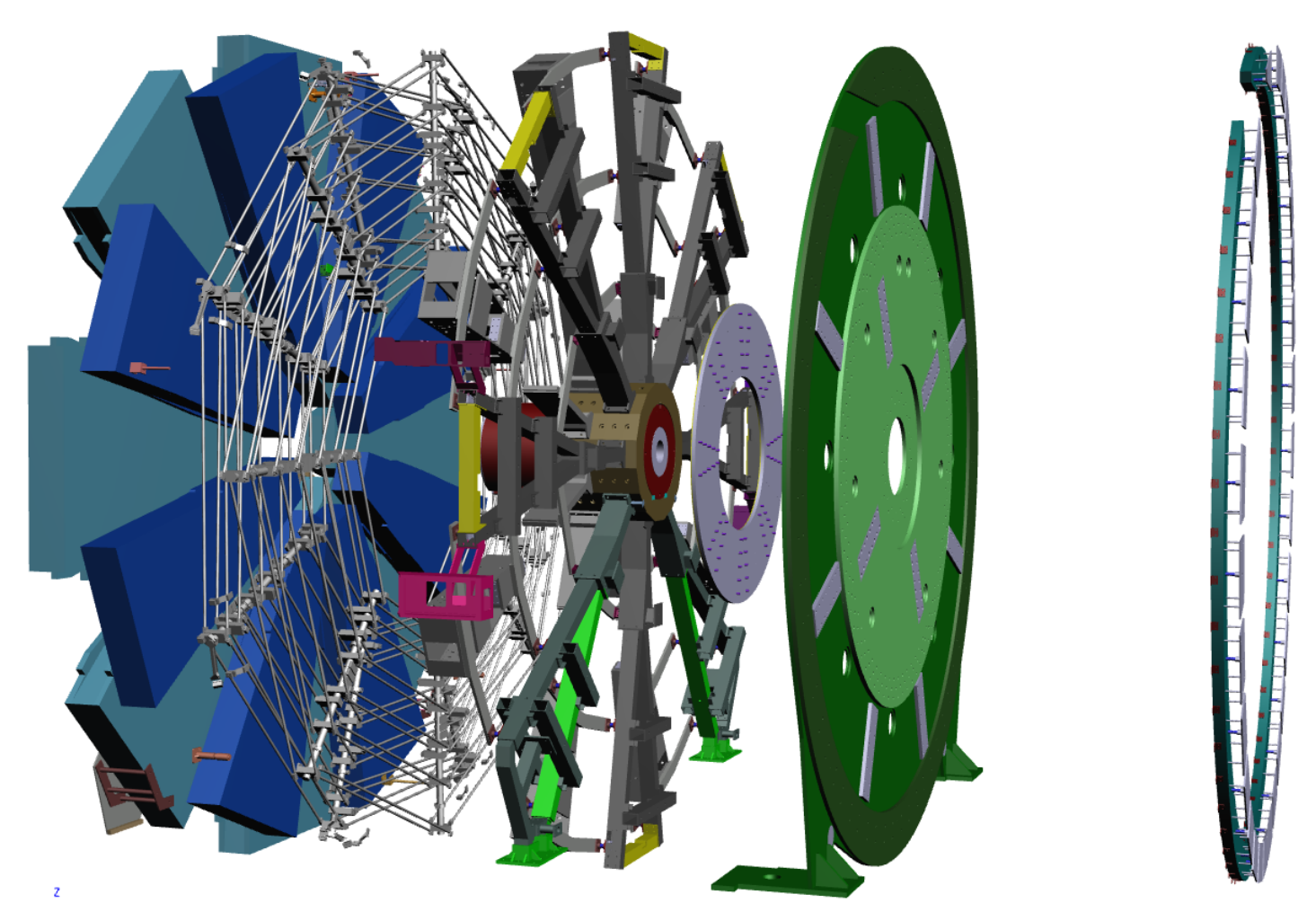

